\title{
TITLE:
}

\section{A micromagnetic study of domain structure modeling}

AUTHOR(S):

Matsuo, Tetsuji; Mimuro, Naoki; Shimasaki, Masaaki

CITATION:

Matsuo, Tetsuji ...[et al]. A micromagnetic study of domain structure modeling. Journal of Magnetism and Magnetic Materials 2008, 320(20): e1029-e1033

ISSUE DATE:

2008-10

URL:

http://hdl.handle.net/2433/85168

RIGHT:

Copyright (C) 2008 Elsevier B.V.; この論文は出版社版でありません。引 用の際には出版社版をご確認ご利用ください。; This is not the published version. Please cite only the published version. 


\title{
A micromagnetic study of domain-structure modeling
}

\author{
Tetsuji Matsuo $^{\mathrm{a} *}$, Naoki Mimuro ${ }^{\mathrm{a}}$, Masaaki Shimasaki ${ }^{\mathrm{b}}$ \\ ${ }^{a}$ Kyoto University, Kyoto 615-8510, Japan \\ ${ }^{b}$ Fukui University of Technology, Fukui 910-8505, Japan
}

\begin{abstract}
To develop a mesoscopic model for magnetic-domain behavior, a domain structure model (DSM) was examined and compared with a micromagnetic simulation. The domain structure of this model is given by several domains with uniform magnetization vectors and domain walls. The directions of magnetization vectors and the locations of domain walls are determined so as to minimize the magnetic total energy of the magnetic material. The DSM was modified to improve its representation capability for domain behavior. The domain wall energy is multiplied by a vanishing factor to represent the disappearance of magnetic domain. The sequential quadratic programming procedure is divided into two steps to improve an energy minimization process. A comparison with micromagnetic simulation shows that the modified DSM improves the representation accuracy of the magnetization process.
\end{abstract}

Keywords: Domain structure model; micromagnetic simulation; mesoscopic model; magnetic thin film;

\section{Introduction}

The micromagnetic simulation solving the LLG equation [1] is a powerful tool to describe the microscopic behavior of magnetic materials. However, it is difficult for the micromagnetic simulation to describe mesoscopic or macroscopic magnetic behavior because of its high computational cost. Several domain structure models (DSMs) [2]-[5] have been proposed to represent the mesoscopic behavior of magnetic materials. However, the capability of DSMs has not been sufficiently examined because they have not been compared with the micromagnetic simulation.

This paper modifies a DSM to improve its representation capability for the domain behavior. The modified DSM is compared with the micromagnetic simulation to examine its representation accuracy of the magnetization process.

\section{Domain structure model}

There are several DSMs [2]-[5] having different approaches for magnetostatic energy computation and magnetic energy minimization. This study examines Ref. [5]'s DSM.
The domain structure of all DSMs in Refs. [2]-[5] is given by several domains with uniform magnetization vectors and their boundaries (domain walls). Two-dimensional domain structures are studied in this paper. Fig. 1 illustrates an example of the domain structure where $\boldsymbol{m}_{1}, \ldots, \boldsymbol{m}_{4}$ are normalized magnetization vectors. The directions of magnetization vectors and the locations of domain walls are determined so as to minimize the total magnetic energy of the magnetic material.

The total magnetic energy $E$ is given by

$$
E=E_{\text {ap }}+E_{\text {an }}+E_{\mathrm{w}}+E_{\mathrm{st}}
$$

where $E_{\text {ap }}$ is the Zeeman energy, $E_{\text {an }}$ is the anisotropic energy, $E_{\mathrm{w}}$ is the domain wall energy, and $E_{\mathrm{st}}$ is the magnetostatic energy.

The Zeeman energy is given as

$$
E_{\text {ap }}=-\mu_{0} M d \sum_{i=1}^{N_{\mathrm{d}}} S_{i} \mathbf{m}_{i} \cdot \mathbf{H}_{\text {ap }}
$$




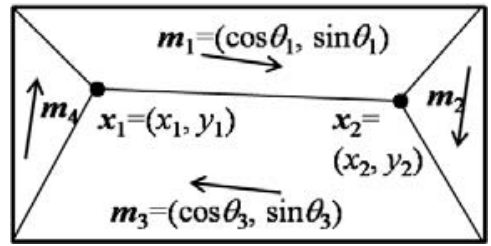

Fig. 1. Domain structure model.

where $M$ is the magnitude of spontaneous magnetization, $d$ is the thickness of the material, $N_{\mathrm{d}}$ is the number of assumed magnetic domains, $S_{i}$ is the area of the $i$-th magnetic domain, and $\boldsymbol{H}_{\mathrm{ap}}$ is the applied magnetic field.

The anisotropic energy is given as

$$
E_{\text {an }}=K_{1} d \sum_{i=1}^{N_{\mathrm{d}}} S_{i} \sin ^{2} \theta_{\mathrm{e} i}
$$

where a uniaxial anisotropy is assumed in this paper, $K_{1}$ is the anisotropy constant, and $\theta_{\mathrm{e} j}$ is the angle between the easy-axis direction and the magnetization vector in the $i$-th domain.

The domain wall energy is given as

$$
E_{\mathrm{w}}=\sum_{j=1}^{N_{\mathrm{w}}} E_{\mathrm{w} j}
$$

where $N_{\mathrm{w}}$ is the number of domain walls and $E_{\mathrm{w} j}$ is the domain wall energy of the $j$-th domain wall. The Neel domain wall [1] is assumed in this study to analyze magnetic thin films. Accordingly, $E_{\mathrm{w} j}$ is given as

$$
E_{\mathrm{w} j}=4 L_{j} d \sqrt{A\left(K_{1}+\frac{\mu_{0} M^{2}}{2}\right)}\left(1-\cos \frac{\theta_{\mathrm{w} j}}{2}\right)^{2}
$$

where $A$ is the exchange stiffness constant, $L_{j}$ is the length of the $j$-th domain wall, and $\theta_{\mathrm{w} j}$ is the angle difference of adjacent magnetization vectors of the $j$-th domain wall.

The magnetostatic energy is determined from the magnetic charges on domain boundaries (domain walls and material boundaries). The magnetic charge density $\sigma_{j}$ on the $j$-th domain wall is given as

$$
\sigma_{j}=\mu_{0} M\left(\boldsymbol{m}_{j 1}-\boldsymbol{m}_{j 2}\right) \cdot \boldsymbol{n}_{j}
$$

where $\boldsymbol{m}_{j 1}$ and $\boldsymbol{m}_{j 2}$ are adjacent normalized magnetization vectors of the $j$-th domain wall and $\boldsymbol{n}_{j}$ is the unit normal vector. For material boundaries, $\boldsymbol{m}_{j 2}=0$. The magnetic scalar potential $\varphi$ is given as

$$
\varphi=\sum_{j=1}^{N_{\mathrm{b}}} \varphi_{j}=\frac{1}{4 \pi \mu_{0}} \sum_{j=1}^{N_{\mathrm{b}}} \int_{\mathrm{W}_{j}} \frac{\sigma_{j}}{r} \mathrm{~d} S
$$

where $N_{\mathrm{b}}$ is the number of domain boundary segments, $\varphi_{j}$ is the magnetic scalar potential given by the $j$-th domain boundary segment $\mathrm{w}_{j}$. The magnetostatic energy is given as

$$
E_{\mathrm{st}}=\frac{1}{2} \sum_{k=1}^{N_{\mathrm{b}}} \int_{\mathrm{W} k} \varphi \sigma_{k} \mathrm{~d} S=\frac{1}{2} \sum_{j=1}^{N_{\mathrm{b}}} \sum_{k=1}^{N_{\mathrm{b}}} \int_{\mathrm{W} k} \varphi_{j} \sigma_{k} \mathrm{~d} S
$$

The magnetic scalar potential $\varphi_{j}$ is given as follows. A rotated coordinate system $(s, t, z)$ is used as illustrated in Fig. 2, where the $s$-axis is parallel to the $j$-th domain boundary segment. The end points of the domain boundary segment are denoted by $\left(s_{j 1}, t_{j}\right)$ and $\left(s_{j 2}, t_{j}\right)$. The potential $\varphi_{j}$ is given as

$$
\begin{aligned}
\varphi_{j}(s, t, z)= & \frac{\sigma_{j}}{4 \pi \mu_{0}} \int_{s j 1}^{s j 2} \int_{0}^{d} \frac{\mathrm{d} z_{j} \mathrm{~d} s_{j}}{\sqrt{\left(s-s_{j}\right)^{2}+\left(t-t_{j}\right)^{2}+\left(z-z_{j}\right)^{2}}} \\
= & \frac{\sigma_{j}}{4 \pi \mu_{0}}\left\{f\left(s-s_{j 1}, t-t_{j}, z-d\right)-f\left(s-s_{j 1}, t-t_{j}, z\right)\right. \\
& \left.-f\left(s-s_{j 2}, t-t_{j}, z-d\right)+f\left(s-s_{j 2}, t-t_{j}, z\right)\right\}
\end{aligned}
$$

where function $f(x, y, z)$ is given by

$$
\begin{aligned}
f(x, y, z)= & y \tan ^{-1} \frac{x z}{y \sqrt{x^{2}+y^{2}+z^{2}}}+z \ln \left(\sqrt{x^{2}+y^{2}+z^{2}}-x\right) \\
& +x \ln \left(\sqrt{x^{2}+y^{2}+z^{2}}-z\right)
\end{aligned}
$$

The integration of $\varphi_{j}$ on the $k$-th domain boundary segment in Eq. (8) is performed analytically along the $z$-direction and numerically along the other direction.

The sequential quadratic programming (SQP) [6] method is used to determine the positions of endpoints of domain walls and the direction of normalized magnetization vectors that give a local minimum of total magnetic energy. To avoid the direct computation of Hessian matrix, the BFGS (BroydenFletcher-Goldfarb-Shanno) scheme [6] is used in the SQP, which is an efficient quasi-Newton scheme. Geometric constraints are imposed for the endpoints of domain walls.

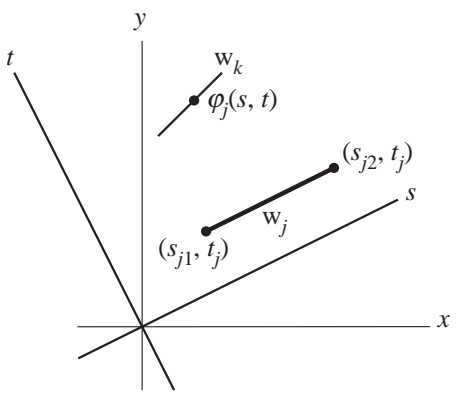

Fig. 2. Coordinate system for magnetic scalar potential.

\section{Improvement of domain structure model}

As is shown later, the DSM described above sometimes fails to represent magnetization processes of magnetic materials. Accordingly, this study modifies the DSM as below. The original and modified DSMs are called "SDSM" (simple DSM) and "MDSM" hereafter.

\subsection{Vanishing factor for domain disappearance}

When the position of a magnetic domain wall coincides with a material boundary because of domain wall movement, the domain between them disappears as is shown in Fig. 3. The disappearance of domain implies the vanishing of the domain wall. However, the domain wall energy of Eq. (5) may remain if the energy minimization procedure fails to decrease 
$\theta_{\mathrm{w} j}$ to zero. To avoid this residual domain wall energy, the domain wall energy $E_{\mathrm{w} j}$ is multiplied by a vanishing factor $v_{j}$ defined as

$$
v_{j}=v\left(\alpha_{j 1}\right) v\left(\alpha_{j 2}\right), \quad v(\alpha)=\left\{\begin{array}{cc}
1 & (\beta \leq \alpha) \\
1-\left(1-\frac{\alpha}{\beta}\right)^{2} & (0 \leq \alpha<\beta)
\end{array}\right.
$$

where $\alpha_{j i}=S_{j i} / l_{j i}(i=1,2)$ are defined for the two adjacent domains to the $j$-th domain wall; $l_{j i}$ is the perimeter of domain $j i$, and $S_{j i}$ is its area; $\beta$ is a constant to give the domain size at which the vanishing factor begins to work. An index of domain width is given by $\alpha_{j i}$ even for a complexly shaped domain.

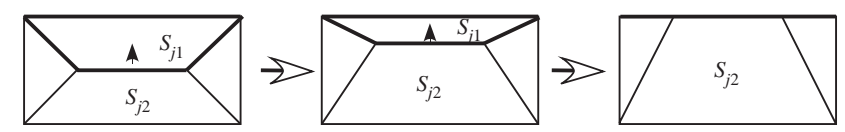

Fig. 3. Disappearance of magnetic domain.

\subsection{Two-step $S Q P$}

The SQP sometimes fails to minimize $E$ because the quadratic approximation using the BFGS formula does not always work well for the mixed variables of domain-wall endpoint positions and magnetization angles under geometric constraints of endpoint positions.

On the other hand, in an actual magnetization process, the domain wall movement usually occurs before the magnetization rotation proceeds.

Accordingly, to improve the energy minimization process, this study divides the SQP procedure into two steps; the domain wall positions are firstly optimized and the magnetization directions are optimized secondly.

\section{Comparison with micromagnetic simulation}

Four types of magnetic thin films with uniaxial anisotropy below are examined:

(IA) $8 \mu \mathrm{m}$ (easy axis direction) $\times 4 \mu \mathrm{m} \times 20 \mathrm{~nm}$,

(IB) $16 \mu \mathrm{m}$ (easy axis direction) $\times 8 \mu \mathrm{m} \times 20 \mathrm{~nm}$,

(IIA) $2 \mu \mathrm{m} \times 1 \mu \mathrm{m}$ (easy axis direction) $\times 20 \mathrm{~nm}$, and

(IIB) $8 \mu \mathrm{m} \times 4 \mu \mathrm{m}$ (easy axis direction) $\times 20 \mathrm{~nm}$.

A back-and-forth magnetic field is applied with an amplitude of $8 \times 10^{4} \mathrm{~A} / \mathrm{m}$ along the direction of the longest edge. $M=1.0 \mathrm{~T}$ and $A=1.3 \times 10^{-11} \mathrm{~J} / \mathrm{m}$ are used for the simulation whereas $K_{1}$ is set at $5.0 \times 10^{2} \mathrm{~J} / \mathrm{m}^{3}$ for films (IA) and (IB), and at $5.0 \times 10^{4} \mathrm{~J} / \mathrm{m}^{3}$ for (IIA) and (IIB).

Micromagnetic simulations are executed by solving the LLG equation using unit cells of $15.625 \mathrm{~nm} \times 15.625 \mathrm{~nm} \times$ $10 \mathrm{~nm}$ for films (IA), (IIA) and (IIB), and a unit cell of $31.25 \mathrm{~nm} \times 31.25 \mathrm{~nm} \times 10 \mathrm{~nm}$ for film (IB).
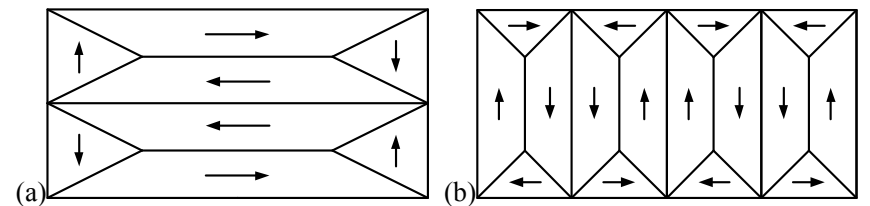

Fig. 4. Assumed domain structures; (a) for films (IA) and (IB), and (b) for films (IIA) and (IIB).

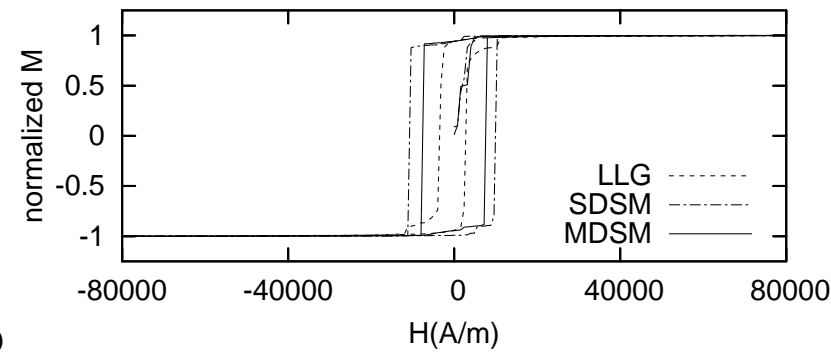

(a)

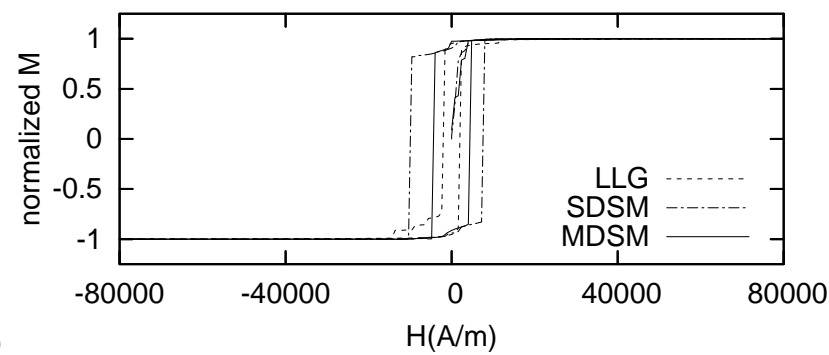

Fig. 5. Averaged MH loops along the applied field direction; (a) film (IA) and (b) film (IB).

\subsection{Simulation with applied field along easy axis}

The SDSM and MDSM are examined for films (IA) and (IB) where the domain structure shown by Fig. 4(a) is assumed.

Fig. 5 shows averaged MH loops along the easy axis direction that are given by the DSMs and the micromagnetic simulation. The comparison shows that the MDSM yields more accurate MH loops than the SDSM. Fig. 6 illustrates the domain structures of film (IB) obtained by the MDSM. Fig. 7 depicts those given by the micromagnetic simulation where brightness shows the perpendicular component of magnetization to the applied field direction. The domain structure obtained by the MDSM qualitatively agrees with that given by the micromagnetic simulation. Further improvement of the MDSM will be required to obtain quantitative agreement with the micromagnetic simulation.

A direct comparison of computational time is not easy between the micromagnetic simulation and the DSMs because the former was executed by a supercomputer and the latter were executed by a PC. The computational time for the DSM is not much affected by the analyzed material size if the same domain structure is assumed. In contrast, the computation time for the micromagnetic simulation rapidly increases with an increase in the number of cells. For example, it is estimated that the micromagnetic simulation for film (IB) requires about 200 times more computation time than the DSM. 
(a)
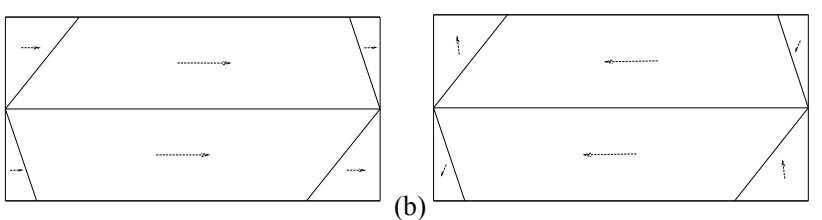

Fig. 6. Domain structures obtained by MDSM; (a) $H_{\text {ap }}=8 \times 10^{4} \mathrm{~A} / \mathrm{m}$ and (b) $H_{\text {ap }}=1.6 \times 10^{3} \mathrm{~A} / \mathrm{m}$ (when $H_{\text {ap }}$ is increasing).

\section{(a)}

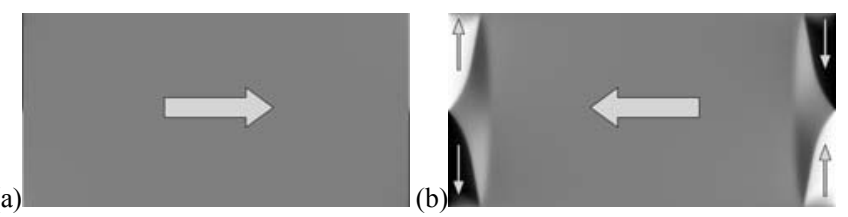

Fig. 7. Magnetic domains given by the micromagnetic simulation; (a) $H_{\text {ap }}=$ $8 \times 10^{4} \mathrm{~A} / \mathrm{m}$ and (b) $H_{\text {ap }}=1.6 \times 10^{3} \mathrm{~A} / \mathrm{m}$ (when $H_{\text {ap }}$ is increasing).

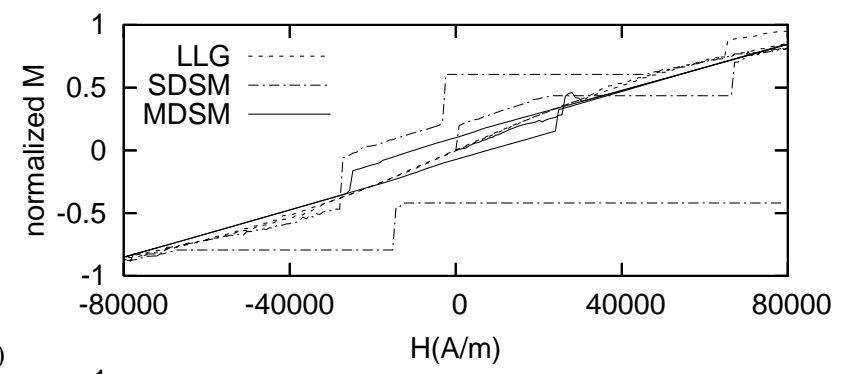

(a)

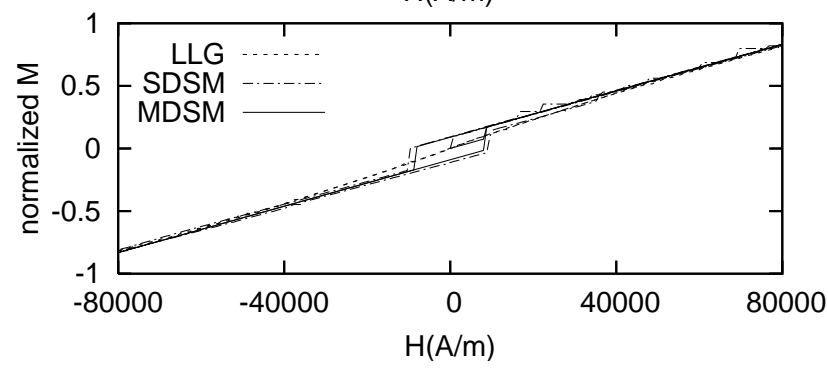

Fig. 8. Averaged $\mathrm{MH}$ loops along the applied field direction; (a) film (IIA) and (b) film (IIB).

\subsection{Simulation with applied field perpendicular to easy axis}

The DSMs are examined for films (IIA) and (IIB) where the domain structure shown by Fig. 4(b) is assumed.

Fig. 8 shows averaged $\mathrm{MH}$ loops along the applied-field direction. Fig. 8(a) shows that the SDSM fails to represent magnetization reversal for film (IIA) whereas the MDSM gives accurate $\mathrm{MH}$ curve. Fig. 9 illustrates the domain structures of film (IIB) obtained by the MDSM whereas Fig. 10 depicts those yielded by the micromagnetic simulation. The former qualitatively agrees with the latter. Figs. 9 and 10 show that triangle domains assumed in Fig. 4(b) disappear, where the SDSM yields excess domain wall energy without the vanishing factor.
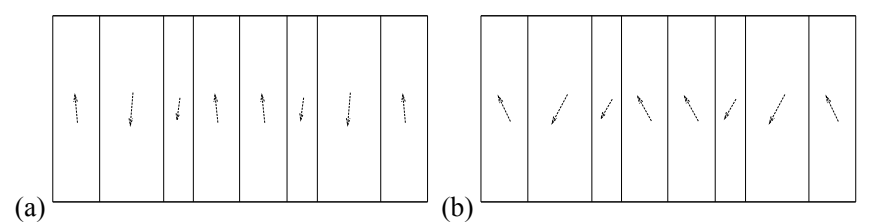

Fig. 9. Domain structures obtained by MDSM; (a) $H_{\text {ap }}=0 \mathrm{~A} / \mathrm{m}$ and (b) $H_{\text {ap }}=$ $-4 \times 10^{4} \mathrm{~A} / \mathrm{m}$.

(a)

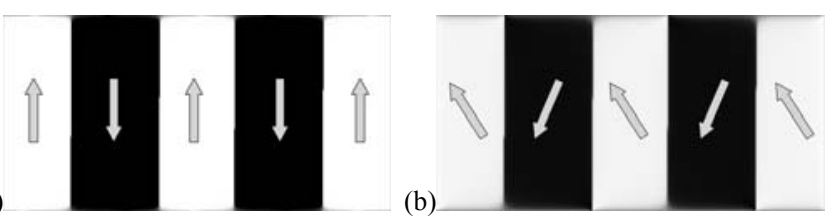

Fig. 10. Magnetic domains given by the micromagnetic simulation; (a) $H_{\text {ap }}=0$ $\mathrm{A} / \mathrm{m}$ and (b) $H_{\mathrm{ap}}=-4 \times 10^{4} \mathrm{~A} / \mathrm{m}$.

\section{Concluding remarks}

This paper modifies a domain structure model (DSM) to improve its representation capability for domain behavior. A comparison with micromagnetic simulation shows that the modification effectively improves the representation accuracy of the magnetization process. The DSM is more efficient than the micromagnetic simulation when the analyzed material size becomes large.

\section{Acknowledgments}

This work was supported in part by the Iwatani Naoji Foundation's Research Grant and by the Japan Society for the Promotion of Science, Grant-in-Aid for Scientific Research (C), 19560288.

\section{References}

[1] H. Kronmüller, M. Fähnle, Micromagnetism and the Microstructure of Ferromagnetic Solids, Cambridge University Press, 2003.

[2] N. Smith, IEEE Trans. Magn. 24 (1988) 2380.

[3] C. Saka, K. Siiki, K. Shinagawa, J. Appl. Phys. 68 (1990) 263.

[4] M. Enokizono, T. Todaka, Y. Midou, IEEE Trans. Magn. 32 (1996) 1172.

[5] T. Matsuo, H. Sugino, M. Shimasaki, Papers Technical Meeting IEE Japan MAG-05-108 (2005).

[6] J.F. Bonnans, J.C. Gilbert, C. Lemaréchal, C.A. Sagastizábal, Numerical Optimization: Theoretical and Practical Aspects, 2nd Ed., SpringerVerlag, Berlin, 2006. 